\title{
Efficient non-volatile holographic recording in doubly doped lithium niobate
}

\section{Karsten Buse, Ali Adibi and Demetri Psaltis}

Department of Electrical Engineering, Mail Stop 136-93, California Institute of Technology, Pasadena, CA 91125, USA

Received 9 September 1998, in final form 17 November 1998

Abstract. We present an efficient, non-volatile holographic storage scheme in doubly doped lithium niobate, with red recording light and simultaneous illumination by homogeneous ultraviolet light.

Keywords: Volume holographic data storage, photorefractive materials, lithium niobate

\section{Introduction}

Photorefractive materials are of particular interest for readwrite holographic data storage. One of the main problems which makes the practical implementation of these memories difficult is the erasure of stored information during readout. Several solutions, such as thermal fixing, electrical fixing and two-step recording, have been proposed for this problem [1-3], but they have some special requirements such as heating the crystal, using high electric fields or high light intensities.

\section{Non-volatile recording}

We present here a simple and practical solution to the erasure problem. The idea is to use two different deep traps, for example iron and manganese in lithium niobate. The energy band diagram for such a system is depicted in figure 1 . The electrons are initially in the deeper traps. Light of short wavelength (with high-energy photons) can transfer some of these electrons to the shallower traps via the conduction band. This makes the recording of the holograms using longer wavelength light feasible. The final hologram is recorded in the deeper traps and, therefore, is not erased during the read-out by the recording (long-wavelength) light. For efficient holographic recording, the electron transfer should be considerable. Doubly doped $\mathrm{LiNbO}_{3}$ crystals are excellent candidates for this recording scheme since they show strong photochromic effects [4], i.e. absorption changes under illumination, which are attributed to the redistribution of a considerable amount of electronic charge between centres of different absorption.

\section{Experiments}

We performed experiments with a $0.85 \mathrm{~mm}$ thick $\mathrm{LiNbO}_{3}$ crystal, doped with 0.075 wt $\% \mathrm{Fe}_{2} \mathrm{O}_{3}$ and 0.01 wt $\%$

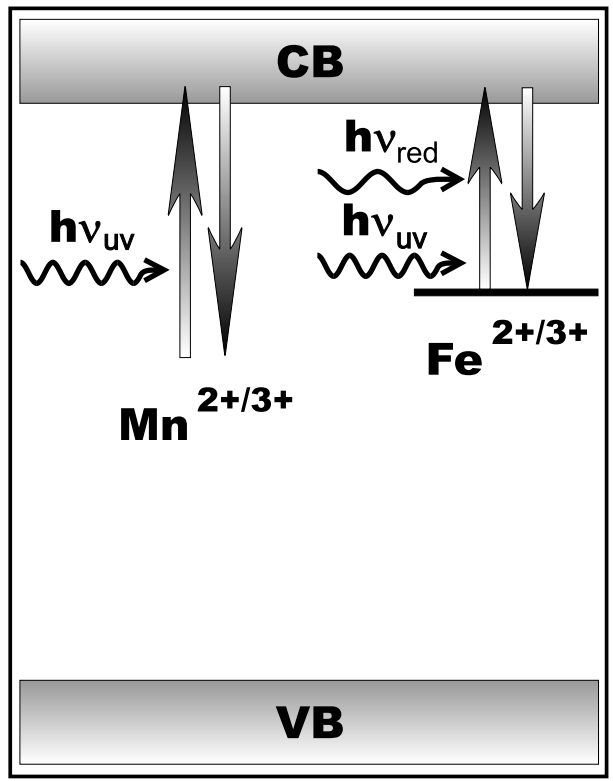

Figure 1. Energy band diagram for a doubly doped lithium niobate crystal.

$\mathrm{MnO}$. The crystal is sensitized for recording by homogeneous illumination with $365 \mathrm{~nm}$ light from a $100 \mathrm{~W}$ mercury lamp. The intensity of this illumination is $0.02 \mathrm{~W} \mathrm{~cm}^{-2}$. The recording beams are two plane waves at $633 \mathrm{~nm}$ with equal intensities of $0.3 \mathrm{~W} \mathrm{~cm}^{-2}$. The read-out is performed by one of these beams, while the other one is blocked. The recording and read-out beams are all ordinarily polarized.

Initial illumination with homogeneous ultraviolet light sensitizes the crystal by transferring some of the electrons from the manganese sites to the iron traps. If we then record the hologram using red beams without simultaneous ultraviolet illumination, the final diffraction efficiency is very small, as shown in figure 2. This is due to the total bleaching 


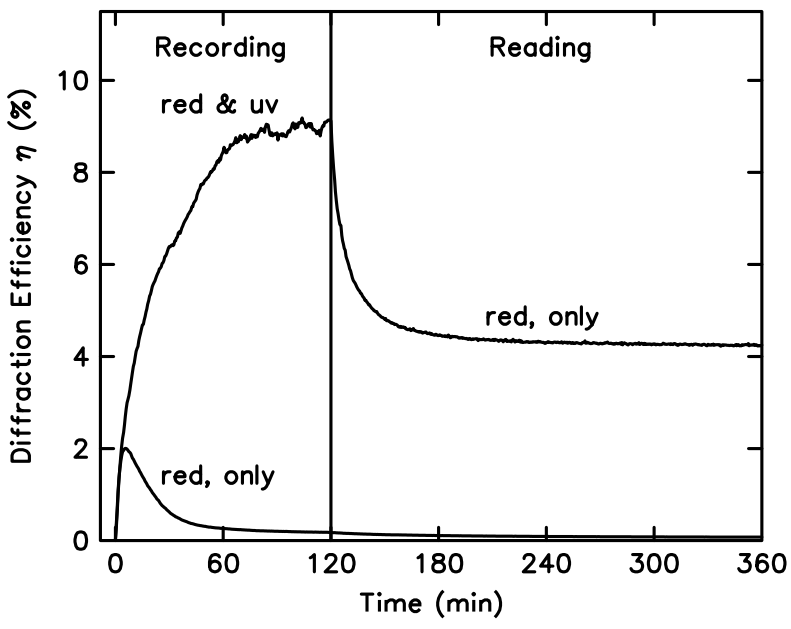

Figure 2. Holographic recording and read-out curves for doubly doped lithium niobate.

of the crystal, i.e. at first the electrons in the iron traps are transferred to the manganese centres in the high-intensity regions, and then the same thing occurs for the low-intensity regions. Therefore, all electrons move by approximately the same distance and are re-trapped by manganese centres. This results in a very small space-charge field and diffraction efficiency. However, if we keep the ultraviolet light on during recording, re-excitation of the electrons trapped in the manganese centres would be possible. This results in higher diffraction efficiencies. Finally, we block the ultraviolet light and illuminate the crystal with a homogeneous red beam to transfer the electrons from iron traps to the manganese centres. This, in fact, reduces the diffraction efficiency of the hologram, but the final hologram cannot be erased by the read-out at red, since it is stored in the manganese centres. This is shown in figure 2 .

If we use extraordinary polarization for the read-out beam, we can achieve a final diffraction efficiency of $32 \%$. This is due to the larger corresponding electro-optic coefficient. Besides the good diffraction efficiency, this method does not suffer from holographic scattering and screening fields due to the presence of the ultraviolet light.

\section{Conclusions}

A theoretical analysis indicates that the final diffraction efficiency depends only on the ratio of the intensities of the sensitizing (ultraviolet) and recording (red) beams and not on the absolute intensities. Depending on the traps, and their corresponding doping levels, there is an optimum value for this intensity ratio which results in the maximum achievable diffraction efficiency.

\section{References}

[1] Amodei J J and Staebler D L 1971 Holographic pattern fixing in electrooptic crystals Appl. Phys. Lett. 18 540-2

[2] Micherson F and Bismuth G 1972 Electrical control of fixation and erasure of holographic patterns in ferroelectric materials Appl. Phys. Lett. 20 79-81

[3] von der Linde D, Glass A and Rodgers K F 1974 Multiphoton photorefractive processes for optical storage in $\mathrm{LiNbO}_{3}$ Appl. Phys. Lett. 25 155-7

[4] Staebler D L and Phillips W 1974 Hologram storage in photochromic $\mathrm{LiNbO}_{3}$ Appl. Phys. Lett. 24 268-70 